\title{
ON POLYNOMIAL APPROXIMATION WITH DEVIATIONS IN PRESCRIBED RATIOS
}

\author{
ABRAHAM SPITZBART AND NATHANIEL MACON ${ }^{1}$
}

1. Introduction. The standard formulas for polynomial interpolation provide a representation for the (unique) polynomial $y(x)$ of degree $n$ such that

$$
y\left(x_{i}\right)=y_{i}, \quad i=0,1, \cdots, n,
$$

where $x_{i}$ and $y_{i}$ are given, with the $x_{i}$ distinct. (For a discussion of these methods, cf. [1]). The present paper considers an extended problem for which a polynomial of degree $n-1$ approximates the $n+1$ values $y_{i}$ at $x=x_{i}$, respectively, with deviations from the given values which are in prescribed ratios. Complete results are obtained for this extended problem, with arbitrary, distinct $x_{i}$.

The results are then applied to the two important special cases (1) equally spaced points (2) a distribution of $x_{i}$ determined by a Chebychev approximation.

2. Statement of the problem. Let $x_{i}, y_{i}, \lambda_{i},(i=0,1, \cdots, n)$ be given, with the $x_{i}$ distinct, throughout. We seek a polynomial $y(x)$ of degree $n-1$ such that

$$
y\left(x_{i}\right)=y_{i}-\lambda_{i} d, \quad(i=0,1, \cdots, n),
$$

where $d$ remains to be determined.

If we write

$$
y(x)=\sum_{\nu=0}^{n-1} b_{\nu} x^{\nu}
$$

then the condition (1) becomes

$$
\sum_{\nu=0}^{n-1} b_{\nu} x_{i}^{\nu}+\lambda_{i} d=y_{i}, \quad(i=0,1, \cdots, n) .
$$

This is a system of $n+1$ linear equations in the $n+1$ quantities $b_{\nu},(\nu=0,1, \cdots, n-1)$, and $d$. Let $B$ denote the column vector consisting of the $b_{v}$ and $d$; let $W$ be the matrix of coefficients of these quantities; and let $Y$ denote the column vector of $y$ 's. In matrix form the system (3) becomes

Received by the editors August 15, 1957.

1 The authors are now at the University of Wisconsin-Milwaukee and the Alabama Polytechnic Institute, respectively. 


$$
W B=Y .
$$

Before proceeding with the solution of this system, we prove a general theorem from which a simple condition that $W$ be nonsingular follows. A result in Polya and Szegö [2] is the special case of this theorem in which $\lambda_{0}=1, \lambda_{i}=0$ for $i=1, \cdots, n$.

THEOREM 1. Let

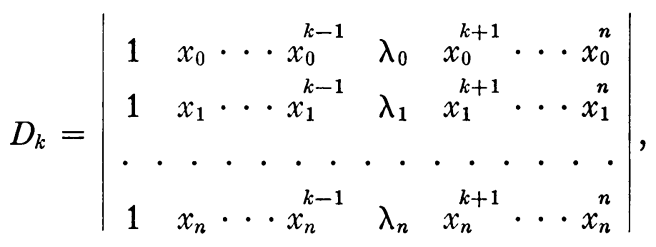

and let $\Delta_{n+1}$ be the Vandermonde determinant obtained by replacing $\lambda_{i}$ by $x_{i}^{k},(i=0,1, \cdots, n)$, in $D_{k}$. Then we have

$$
D_{k}=\Delta_{n+1} \sum_{i=0}^{n} \frac{\lambda_{i}}{p^{\prime}\left(x_{i}\right)} \sum_{j=0}^{n-k}(-1)^{j} \sigma_{j} x_{i}^{n-k-j},
$$

where $p(x)=\prod_{i=0}^{n}\left(x-x_{i}\right)$ and $\sigma_{j}$ is the sum of all products of the $x_{i}$ taken $j$ at a time without repetitions or permutations, $\left(\sigma_{0} \equiv 1\right)$.

Proof. Let $\pi_{n}(x)=\sum_{\nu=0}^{n} c_{\nu} x^{\nu}$ be the polynomial of degree $n$ such that $\pi_{n}\left(x_{i}\right)=\lambda_{i},(i=0,1, \cdots, n)$. If we solve the system

$$
\sum_{\nu=0}^{n} c_{\nu} x_{i}^{\nu}=\lambda_{i}, \quad i=0,1, \cdots, n,
$$

by Cramer's rule, it follows that $c_{k}=D_{k} / \Delta_{n+1}$. But $c_{k}$ can also be obtained by applying Lagrange's interpolation formula to obtain $\pi_{n}(x)$ and then collecting the terms involving $x^{k}$, which gives for $c_{k}$ the double sum in the statement of the theorem; the theorem is proved. In particular, we have $c_{n}=\sum_{i=0}^{n} \lambda_{i} / p^{\prime}\left(x_{i}\right)$.

Setting $k=n$ we obtain the following

Corollary.

$$
\text { Det } W=\Delta_{n+1} \sum_{i=0}^{n} \frac{\lambda_{i}}{p^{\prime}\left(x_{i}\right)}=\Delta_{n+1} c_{n} .
$$

Since the $x_{i}$ are assumed to be distinct, we have $\Delta_{n+1} \neq 0$. Thus, the following theorem holds.

THEOREM 2. The system (3) is nonsingular if and only if $c_{n} \neq 0$, i.e., if and only if no polynomial of degree less than $n$ passes through the points $\left(x_{i}, \lambda_{i}\right), i=0,1, \cdots, n$. 
For later applications we state the result below.

Corollary. If the $x_{i},(i=0,1, \cdots, n)$, are monotonic, and the $\lambda_{i}$ are alternating in sign, then $W$ is nonsingular.

3. Determination of the deviations. We now assume that the condition of Theorem 2 is satisfied. We may then write the solution of (3) as

$$
B=W^{-1} Y \text {. }
$$

The solution of this system depends on the determination of $W^{-1}$, which inverse is independent of the $y_{i}$. Let $w_{i j}(i, j=1,2, \cdots, n+1)$ denote the element in the $i$ th row and $j$ th column of $W^{-1}$. Since $d$, uniquely determined by (3), is the last element in the column vector $B$, it follows that

$$
d=\sum_{j=0}^{n} w_{n+1, j+1} y_{j}
$$

Further, we can prove

TheOREM 3. The elements in the last row of $W^{-1}$ are given by

$$
w_{n+1, j+1}=\frac{1}{c_{n} p^{\prime}\left(x_{j}\right)},
$$

$j=0,1, \cdots, n$. Thus, the ratios of the elements in the last row of $W^{-1}$ are completely determined by the distribution of the $x_{i}$, and are independent of the $\lambda_{i}$.

Proof. Rewriting (3) as $\sum_{v=0}^{n-1} b_{v} x_{i}^{\nu}=y_{i}-\lambda_{i} d,(i=0,1, \cdots, n)$, one sees that the polynomial $y(x)$ defined by (2) takes on the $n+1$ values $y_{i}-\lambda_{i} d$ at the $x_{i},(i=0,1, \cdots, n)$. Therefore, by Lagrange's formula, it may be written

$$
y(x)=\sum_{i=0}^{n} \frac{p(x)}{p^{\prime}\left(x_{i}\right)\left(x-x_{i}\right)}\left(y_{i}-\lambda_{i} d\right) .
$$

Since, on the other hand, $y(x)$ is of degree $n-1$, the coefficient of $x^{n}$ in this sum is zero; thus,

$$
\sum_{i=0}^{n} \frac{y_{i}-\lambda_{i} d}{p^{\prime}\left(x_{i}\right)}=0
$$

Consequently, we have 


$$
d=\frac{1}{c_{n}} \sum_{i=0}^{n} \frac{y_{i}}{p^{\prime}\left(x_{i}\right)} .
$$

Since the elements $w_{n+1, j+1}$ are independent of the $y_{i}$, the result follows immediately by comparing the above with (6).

The polynomial (7) along with the value of $d$ given by (8) constitutes one form of the polynomial $y(x)$ sought in the original statement of the problem. Should we require the individual coefficients $b_{\nu}$ $(\nu=0,1, \cdots, n-1)$, the following methods are available:

(i) We may substitute the now known value of $d$ into any $n$ of the equations (3) and solve the resulting system. The matrix of coefficients is a Vandermonde matrix, the inverse of which is known explicitly [3];

(ii) We may collect terms of the various powers of $x$ in (7);

(iii) We may obtain an explicit representation for $W^{-1}$ and perform the indicated matrix multiplication in (5).

We choose to develop method (iii) in detail, since the determination of $W^{-1}$ is of interest in itself.

4. Inversion of the matrix of coefficients. The elements in the last row of $W^{-1}$ are given by Theorem 3. In this paragraph, we show that the remaining elements of $W^{-1}$ can be expressed very simply in terms of the elements in this last row and the elements of the inverse of the Vandermonde matrix $V=\left\{x_{i-1}^{j-1}\right\},(i, j=1,2, \cdots, n+1)$ of order $n+1$ [cf. 3].

Again we assume $c_{n} \neq 0$, and rewrite (4) in the form

$$
-\frac{1}{c_{n}}\left(\sum_{\nu=0}^{n-1} c_{\nu} x_{i}^{\nu}-\lambda_{i}\right)=x_{i}^{n} \text {. }
$$

Thus, if we write

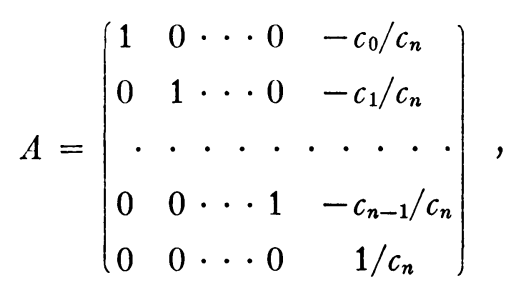

it follows that

$$
W A=V
$$

and so

$$
W^{-1}=A V^{-1}
$$


Let us denote the elements of $V^{-1}$ by $v_{i j}(i, j=1,2, \cdots, n+1)$. Performing the matrix multiplication indicated above, we get

$$
\begin{aligned}
w_{n+1, j} & =v_{n+1, j} / c_{n}, \\
w_{i j} & =v_{i j}-\frac{c_{i-1}}{c_{n}} v_{n+1, j}=v_{i j}-c_{i-1} w_{n+1, j},
\end{aligned}
$$

for $i=1,2, \cdots, n ; j=1,2, \cdots, n+1$. Finally, it follows from (4) that

$$
c_{i-1}=\sum_{j=1}^{n+1} v_{i j} \lambda_{j-1},
$$

for $i=1,2, \cdots, n+1$. We shall call the quantity $c_{i-1}$ the $\lambda$-sum of the ith row of $V^{-1}$.

The results are summarized below.

THEOREM 4. The elements of $W^{-1}$ can be obtained from those of $V^{-1}$ as follows:

(a) the elements of the last row are given by $w_{n+1, j}=v_{n+1, j} / c_{n}$, $(j=1,2, \cdots, n+1)$;

(b) to obtain the ith row of $W^{-1}$ for $i \leqq n$, form the $\lambda$-sum of the ith row of $V^{-1}$ and subtract the product of this $\lambda$-sum and the last row of $W^{-1}$ from the ith row of $V^{-1}$.

Now let $k$ be any of the numbers $0,1, \cdots, n$, and assume $c_{k} \neq 0$. Let $\left\{D_{k}\right\}$ denote the matrix of the $D_{k}$ displayed in Theorem 1 , and let $d_{i j}$ be the element in the $i$ th row and $j$ th column of $\left\{D_{k}\right\}^{-1}$. By methods similar to those used above, one can derive the relations

$$
\begin{aligned}
d_{k+1, j} & =v_{k+1, j} / c_{k}, \\
d_{i j} & =v_{i j}-\frac{c_{i-1}}{c_{k}} v_{k+1, j}=v_{i j}-c_{i-1} d_{k+1, j},
\end{aligned}
$$

where $i=1,2, \cdots, k, k+2, \cdots, n+1 ; j=1,2, \cdots, n+1$. Clearly, Theorem 4 is the special case in which $k=n$.

5. Application to equally spaced points. Suppose now that $x_{i}$ $=x_{0}+i h,(i=0,1, \cdots, n)$. We show that in this case the elements of the last row of $W^{-1}$ are simple to obtain from Theorem 3; and thus, the remaining rows come immediately from Theorem 4 and a previous result expressing the elements of $V^{-1}$ in terms of Stirling numbers when the $x_{i}$ are equally spaced [3].

We have, from Theorem 4,

$$
w_{n+1, j+1}=1 /\left[c_{n} p^{\prime}\left(x_{j}\right)\right],
$$


$(j=0,1, \cdots, n)$, where $p(x)=\prod_{i=0}^{n}\left(x-x_{i}\right)$, and $c_{n}=\sum_{i=0}^{n} \lambda_{i} / p^{\prime}\left(x_{i}\right)$ is assumed to be nonzero. Since here, $x_{j}-x_{i}=(j-i) h$, one sees that

$$
p^{\prime}\left(x_{j}\right)=(-1)^{n-j} j !(n-j) ! h^{n}=(-1)^{n-i} n ! h^{n} /\left(\begin{array}{l}
n \\
j
\end{array}\right) .
$$

Hence, we have

Theorem 5. If the points $x_{i}(i=0,1, \cdots, n)$ are equally spaced, then the elements of the last row of $W^{-1}$ are proportional to the binomial coefficients; more exactly, if $x_{i}=x_{0}+i h$, then

$$
w_{n+1, j+1}=(-1)^{n-j}\left(\begin{array}{l}
n \\
j
\end{array}\right) /\left(n ! h^{n} c_{n}\right), \quad(j=0,1, \cdots, n)
$$

where $c_{n}=\sum_{i=0}^{n} \lambda_{i} / p^{\prime}\left(x_{i}\right)$ is assumed to be nonzero.

As an illustration in which $c_{n}$ may be easily evaluated, we choose the important special case for which $\lambda_{i}=(-1)^{i}, i=0,1, \cdots, n$. We know from the corollary to Theorem 2 that $W^{-1}$ exists for this case. We have

$$
c_{n}=\sum_{i=0}^{n}(-1)^{i} / p^{\prime}\left(x_{i}\right)=\left[(-1)^{n} /\left(n ! h^{n}\right)\right] \sum_{i=0}^{n}\left(\begin{array}{c}
n \\
i
\end{array}\right)=(-1)^{n} 2^{n} /\left(n ! h^{n}\right)
$$

and, further,

$$
w_{n+1, j+1}=(-1)^{n}\left(\begin{array}{c}
n \\
i
\end{array}\right) /(2 h)^{n} .
$$

For a given set of $y_{i}$ we may now obtain $d$, as given in (6), explicitly as

$$
d=\left[1 /(2 h)^{2}\right] \sum_{i=0}^{n}(-1)^{i}\left(\begin{array}{l}
n \\
i
\end{array}\right) y_{i}
$$

6. Application to Chebychev approximation. Another important special case of the problem here considered was treated in a recent paper [4]. The points $x_{i}, i=0,1, \cdots, n$ are defined by

$$
x_{0}=0, \quad x_{n}=1, \quad T_{n}^{\prime}\left(x_{i}\right)=0, \quad i=1,2, \cdots, n-1
$$

where $T_{n}(x)$ is the Chebychev polynomial of degree $n$ for the interval $(0,1)$,

$$
T_{n}(x)=(-1)^{n} \cos [n \operatorname{arc} \cos (2 x-1)]
$$

and $\lambda_{i}=(-1)^{i}, i=0,1, \cdots, n$. (For a general discussion of Chebychev approximation see $[5$, p. $197 \mathrm{ff}]$. Numerous examples are given 
in [6], and an application to the determination of optimum interval tables may be found in [7].) In particular, we may write $x_{j}$ explicitly as

$$
x_{j}=\sin ^{2}(j / 2 n) \pi, \quad j=0,1, \cdots, n .
$$

The polynomial $p(x)=\prod_{i=0}^{n}\left(x-x_{i}\right)$ becomes

$$
p(x)=x(x-1) T_{n}^{\prime}(x) /\left(n \cdot 2^{2 n-1}\right)
$$

from which we obtain

$$
\begin{aligned}
p^{\prime}\left(x_{0}\right)=-\frac{T_{n}^{\prime}(0)}{n \cdot 2^{2 n-1}}, \quad p^{\prime}\left(x_{n}\right)=\frac{T_{n}^{\prime}(1)}{n \cdot 2^{2 n-1}}, \quad p^{\prime}\left(x_{i}\right) & =\frac{x_{i}\left(x_{i}-1\right) T_{n}^{\prime}\left(x_{i}\right)}{n \cdot 2^{2 n-1}}, \\
i & =1,2, \cdots, n-1 .
\end{aligned}
$$

From [4], we then obtain

$$
\begin{array}{r}
p^{\prime}\left(x_{0}\right)=n / 2^{2 n-2}, \quad p^{\prime}\left(x_{n}\right)=(-1)^{n} n / 2^{2 n-2}, \quad p^{\prime}\left(x_{i}\right)=(-1)^{i} n / 2^{2 n-1}, \\
i=1,2, \cdots, n-1 .
\end{array}
$$

Here again $c_{n}$ is easily computed, and is $c_{n}=2^{2 n-1}$. The last row of $W^{-1}$ then becomes

$$
\frac{1}{2 n},-\frac{1}{n}, \frac{1}{n}, \ldots, \frac{(-1)^{n-1}}{n}, \frac{(-1)^{n}}{2 n}
$$

and the familiar ratios $1,-2,2, \cdots,(-1)^{n-1} 2,(-1)^{n}$, appear.

\section{REFERENCES}

1. F. B. Hildebrand, Introduction to numerical analysis, New York, McGrawHill, 1956.

2. G. Polya and G. Szegö, Aufgaben und Lehrsätze aus der Analysis, Vol. II, New York, Dover, 1945, Problem 10, p. 99.

3. N. Macon, and A. Spitzbart, Inverses of Vandermonde matrices, Amer. Math. Monthly vol. 65 (1958) pp. 95-100.

4. A. Spitzbart, and D. L. Shell, A Chebycheff fitting criterion, Journal of the Association for Computing Machinery vol. 5 (1958) pp. 22-31.

5. A. S. Householder, Principles of numerical analysis, New York, McGraw-Hill, 1953, pp. 197-200.

6. C. Hastings, Jr. Approximations for digital computers, Princeton University Press, 1955.

7. H. R. J. Grosch, The use of optimum interval mathematical tables, Proceedings Scientific Computation Forum, I.B.M. Corp., New York, 1948, pp. 23-27.

General Electric Company, Cincinnati, Ohio 